\title{
High Resource Utilization Does Not Affect Mortality in Acute Respiratory Failure Patients Managed With Tracheostomy
}

\author{
Bradley D Freeman MD, Dustin Stwalley MA, Dennis Lambert PhD, Joshua Edler MSc, \\ Peter E Morris MD, Sofia Medvedev PhD, Samuel F Hohmann PhD, \\ and Steven M Kymes PhD
}

\begin{abstract}
BACKGROUND: Tracheostomy practice in patients with acute respiratory failure (ARF) varies greatly among institutions. This variability has the potential to be reflected in the resources expended providing care. In various healthcare environments, increased resource expenditure has been associated with a favorable effect on outcome. OBJECTIVE: To examine the association between institutional resource expenditure and mortality in ARF patients managed with tracheostomy. METHODS: We developed analytic models employing the University Health Systems Consortium (Oakbrook, Illinois) database. Administrative coding data were used to identify patients with the principal diagnosis of ARF, procedures, complications, post-discharge destination, and survival. Mean resource intensity of participating academic medical centers was determined using risk-adjusted estimates of costs. Mortality risk was determined using a multivariable approach that incorporated patient-level demographic and clinical variables and institution-level resource intensity. RESULTS: We analyzed data from 44,124 ARF subjects, 4,776 (10.8\%) of whom underwent tracheostomy. Compared to low-resource-intensity settings, treatment in high-resource-intensity academic medical centers was associated with increased risk of mortality (odds ratio $1.11,95 \%$ CI 1.05-1.76), including those managed with tracheostomy (odds ratio high-resource-intensity academic medical center with tracheostomy 1.10, 95\% CI 1.04-1.17). We examined the relationship between complication development and outcome. While neither the profile nor number of complications accumulated differed comparing treatment environments $(P>.05$ for both), mortality for tracheostomy patients experiencing complications was greater in high-resource-intensity $(95 / 313$, $30.3 \%)$ versus low-resource-intensity $(552 / 2,587,21.3 \%)$ academic medical centers $(P<.001)$. CONCLUSIONS: We were unable to demonstrate a positive relationship between resource expenditure and outcome in ARF patients managed with tracheostomy. Key words: tracheostomy; acute respiratory failure; mechanical ventilation; critical illness; practice variation; quality assurance. [Respir Care 2013;58(11):1863-1872. ( 2013 Daedalus Enterprises]
\end{abstract}

\section{Introduction}

Tracheostomy is one of the most commonly performed surgical procedures among patients with acute respiratory

\footnotetext{
Dr Freeman is affiliated with the Department of Surgery; and Mr Stwalley, Dr Lambert, Mr Edler, and Dr Kymes are affiliated with the Center for Economic Evaluation in Medicine, Washington University School of Medicine, St Louis, Missouri. Dr Morris is affiliated with the Department of Pulmonary Medicine, Wake Forest University Health Sciences Center, Wake Forest, North Carolina. Drs Medvedev and Hohmann are affiliated
}

failure (ARF). ${ }^{1-4}$ Though a minority of all individuals requiring ventilatory support, tracheostomy patients place substantial demands on ventilator, ICU, hospital, and post-

\footnotetext{
with University Health Systems Consortium, Oakbrook, Illinois. Dr Hohmann is also affiliated with the Department of Health Systems Management, Rush University, Chicago, Illinois.

Dr Freeman presented a version of this paper at the annual meeting of the United States Critical Illness and Injuries Trials Group, held November 6, 2012, in Bethesda, Maryland.
} 
hospital discharge resources. ${ }^{5-7}$ Financial expenditures to support the care of tracheostomy patients are among the highest of any diagnostic or procedural group. ${ }^{8}$ Efforts to refine tracheostomy practice have the potential to affect both the quality of care provided this segment of the critically ill population, as well as the resources expended delivering this care. ${ }^{9-11}$

See the Related Editorial on Page 1995

Despite decades of experience, the potential advantages of tracheostomy, relative to prolonged translaryngeal intubation, remain poorly defined, as do the risks associated with this procedure. ${ }^{2,9}$ The timing of tracheostomy has been particularly debated. Rodriguez et al reported that tracheostomy performed within the first week of ICU admission following major trauma was associated with significantly shorter duration of mechanical ventilation and ICU stay, compared with tracheostomy performed later in the course of respiratory failure. ${ }^{12}$ Other investigators have confirmed $^{13,14}$ and contradicted ${ }^{15-17}$ the putative benefits of early tracheostomy. Three recently completed randomized controlled trials suggest that the timing of tracheostomy does not affect the incidence of infectious complications, duration of mechanical ventilation, or ICU or hospital stay. ${ }^{18-20}$ Currently, there are no well established standards to guide patient selection or other aspects of this procedure. Absent such standards, tracheostomy appears to be used in, at worst, an arbitrary, or, at best, a variable fashion. .,21,22 $^{2}$

As is the case with critical care practice in general, variation in management of tracheostomy patients has the potential to be reflected in variability in the resources utilized in providing care. ${ }^{23}$ In some healthcare settings, increased resource expenditure appears to be associated with favorable effects on outcome. ${ }^{24-27} \mathrm{We}$ undertook the current investigation to examine the hypothesis that increased resource expenditure associated with the management of tracheostomy patients-controlling for potentially confounding variables-is associated with a beneficial effect on mortality.

This research was partly supported National Institutes of Health grant UL1 RR02499. The authors have disclosed no conflicts of interest.

Supplementary material related to this paper is available at http:// www.rcjournal.com.

Correspondence: Bradley D Freeman MD, Department of Surgery, Washington University School of Medicine, 660 South Euclid Avenue, Box 8109, St Louis MO 63110.

DOI: $10.4187 /$ respcare. 02359

\section{QUICK LOOK}

\section{Current knowledge}

Tracheostomy is one of the most common surgeries in patients with acute respiratory failure. Tracheostomy patients place substantial demands on ICU, hospital, and post-hospital resources. The costs of care for tracheostomy patients are among the highest of any diagnostic or procedural group.

\section{What this paper contributes to our knowledge}

There was no positive relationship between resource expenditure and outcome in tracheostomy patients. It is imperative to identify clinically beneficial strategies related to tracheostomy, including patient selection, timing, technique, and post-procedure management, and to assess the cost-effectiveness of these strategies.

\section{Methods}

\section{Description of Data Resource and Identification of Patients}

This study is based on analysis of the University Health Systems Consortium, (Oakbrook, Illinois) data resource. The University Health Systems Consortium is a network of university-based tertiary care institutions and affiliated hospitals representing $90 \%$ of the nation's non-profit academic medical centers. University Health Systems Consortium has developed a highly detailed administrative database that enables comparative analysis in clinical, operational, financial, and patient safety domains among participants.

Adult patients ( $\geq 18$ years of age) with the principal diagnosis of ARF (International Classification of Diseases, 9th Revision [ICD-9] diagnosis code 518.81) and requiring mechanical ventilation were identified through use of the ICD-9 procedure codes for ventilator support of any duration (96.70 Continuous invasive mechanical ventilation of unspecified duration, 96.71 Continuous invasive mechanical ventilation for less than 96 consecutive hours, 96.72 Continuous invasive mechanical ventilation for 96 consecutive hours or more, 96.7 Other continuous invasive mechanical ventilation). Patients undergoing tracheostomy were identified in a similar fashion (31.1 Temporary tracheostomy).

\section{Risk Adjustment}

The approach to risk adjustment used in this study has been previously described. ${ }^{28-30}$ Briefly, 2 independent mod- 
els (hospital cost and probability of in-patient mortality) were constructed for each diagnosis-related group (DRG) or base Medicare severity DRG. Each model incorporated an all-patient-refined DRG severity of illness or risk of mortality category (severity of illness and risk of mortality each are assigned a score on a 4-point scale of $1=$ "minor" to $4=$ "extreme"), as well as 29 specific comorbidities identified by the Agency for Healthcare Research and Quality as influencing the outcome of interest. ${ }^{31}$

For model development, data were partitioned into derivation and validation data sets and assessed using standard diagnostics (concordance index, Hosmer-Lemeshow test, and $\mathrm{R}^{2}$ ). In the event that it was not possible to develop a stable model by this approach, each case in the DRG or base Medicare severity DRG was assigned the average observed value, stratified first by severity of illness or risk of mortality, and then by transfer status within each severity-of-illness or risk-of-mortality level. Cost of patient care was assessed using the ratio of cost to charges methodology. Detailed charges were collected at the revenue code level and were mapped into departments and cost centers, consistent with the Centers for Medicare and Medicaid Services descriptions. Centers for Medicare and Medicaid Services cost reports were used to obtain service line costs and charges, which were used to calculate a cost-to-charge ratio for each service. In addition, a costto-charge ratio for each cost center within the hospital, as well as a global cost-to-charge ratio for the hospital, was generated. These cost-to-charge ratios were used to estimate percentiles for refining or trimming the data, to eliminate outliers. Estimated service center costs were derived from multiplying charges by the cost-to-charge ratio. Total costs were computed by summing individual cost center estimates. A predicted value of cost and probability of mortality were assigned to each patient record in the data set.

\section{Identification of Complications}

The University Health Systems Consortium has developed and validated methodology to identify 25 specific complications based on diagnosis and procedural codes: postoperative stroke, aspiration pneumonia, postoperative pulmonary compromise, postoperative gastrointestinal hemorrhage or ulceration, postoperative urinary tract complication, cellulitis or decubitis ulcer, septicemia, postoperative/intra-operative shock due to anesthesia, reopening of surgical site, mechanical complication due to device or implant, miscellaneous complications, shock or respiratory arrest, central or peripheral nervous system complication, postoperative acute myocardial infarction, postoperative myocardial abnormality (excluding myocardial infarction), postoperative infections excluding pneumonia or wounds, procedure related perforations or lacerations, postoperative coma or stupor, postoperative pneumonia, postoperative physiologic or metabolic derangements, complications related to anesthetic or central nervous system agents, venous thrombosis/pulmonary embolism, wound infection, post-procedure hemorrhage or hematoma, and other complications of procedures.

\section{Approach to Analysis}

We evaluated the relationship between in-patient mortality, presence of tracheostomy, and resource-intensity using multivariable logistic regression. Resource intensity was operationalized for all ARF patients in a given facility: not for the individual patient. We took this approach because we have previously documented significant interinstitutional differences in tracheostomy practice (in terms of proportion of patients undergoing this procedure, as well as timing) and felt that institution-level resource intensity would reflect this variation. ${ }^{5}$ Average resource intensity was estimated for each facility by dividing total costs associated with providing care by expected cost, as predicted by the University Health Systems Consortium algorithm. We hypothesized that increased resource intensity would result in a beneficial effect on survival, and took 2 approaches to incorporating resource intensity into our analytic model. In the first approach, resource intensity was modeled as a dichotomous variable, such that a facility that consumed $\geq 25 \%$ of predicted resources (ie, a proportional expense ratio $\geq 1.25$ ) was considered high resource intensity, while a facility providing care associated with a proportional expense ratio $<1.25$ was considered low resource intensity. Institutional proportional expense ratio was stable over the years included in this study, so we pooled data over this time period for analysis (see the supplementary materials at http://www.rcjournal.com). Stratifying the institutions into high and low resourceintensity categories has the potential to introduce bias. To compensate for this potential bias, and to more generally examine the relationship between resource expenditure and outcome, we also modeled resource intensity as a continuous variable. Univariate analyses were conducted using logistic regression to identify variables that could confound the relationship between mortality and resource use. In building our multivariable model we controlled for the possibility that the relationship between mortality and tracheostomy might vary by level of resource intensity by including these 2 variables as an interaction term. All analyses were conducted using statistics software (SAS 9.2, SAS Institute, Cary North, Carolina). For ease of presentation, findings presented in the Results section represent the dichotomous model; the supplementary materials at http://www.rcjournal.com present the findings of resource intensity modeled as a continuous variable. 
Table 1. Baseline Characteristics of Adult Subjects With Acute Respiratory Failure Cared for in Academic Medical Centers

\begin{tabular}{|c|c|c|c|c|}
\hline & $\begin{array}{l}\text { All Subjects } \\
n=44,124\end{array}$ & $\begin{array}{c}\text { Non-tracheostomy } \\
\text { Subjects } \\
n=39,348\end{array}$ & $\begin{array}{c}\text { Tracheostomy } \\
\text { Subjects } \\
n=4,776\end{array}$ & $P^{*}$ \\
\hline Age, mean $\pm \mathrm{SD}$ y & $61.3 \pm 21.0$ & $61.4 \pm 19.8$ & $60.4 \pm 13.8$ & $<.001$ \\
\hline Male & $22,635(51.3)$ & $20,146(51.2)$ & $2,478(51.9)$ & .42 \\
\hline Ethnicity & & & & .005 \\
\hline White & $25,680(58.2)$ & $22,782(57.9)$ & $2,870(60.1)$ & \\
\hline African American & $13,237(30.0)$ & $11,096(28.2)$ & $1,232(25.8)$ & \\
\hline Hispanic & $1,941(4.4)$ & $1,770(4.5)$ & $205(4.3)$ & \\
\hline Other & $4,147(9.4)$ & $3,699(9.4)$ & $468(9.8)$ & \\
\hline Admission status & & & & $<.001$ \\
\hline Emergency & $31,857(72.2)$ & $28,763(73.1)$ & $3,109(65.1)$ & \\
\hline Urgent & $10,060(22.8)$ & $8,735(22.2)$ & $1,337(28.0)$ & \\
\hline Elective & $1,676(3.8)$ & $1,416(3.6)$ & $243(5.1)$ & \\
\hline Other or no data & $485(1.1)$ & $393(1.0)$ & $86(1.8)$ & \\
\hline \multicolumn{5}{|l|}{ Comorbidities } \\
\hline Hypertension & $23,165(52.5)$ & $20,893(53.1)$ & $2,306(48.3)$ & $<.001$ \\
\hline Fluid and electrolyte disorders & $21,753(49.3)$ & $18,926(48.1)$ & $2,808(58.8)$ & $<.001$ \\
\hline Chronic pulmonary disease & $21,488(48.7)$ & $19,280(49.0)$ & $2,173(45.5)$ & $<.001$ \\
\hline Congestive heart failure & $15,972(36.2)$ & $14,283(36.3)$ & $1,685(35.3)$ & .19 \\
\hline Diabetes & $13,104(29.7)$ & $11,725(29.8)$ & $1,399(29.3)$ & .51 \\
\hline Anemia & $11,560(26.2)$ & $10,033(25.5)$ & $1,542(32.3)$ & $<.001$ \\
\hline Neurological disorder & 9,177 (20.8) & 7,987 (20.3) & $1,217(25.5)$ & $<.001$ \\
\hline Renal failure & $7,809(17.7)$ & $7,043(17.9)$ & $773(16.2)$ & .005 \\
\hline Psychiatric disorder (depression, psychoses) & $7,015(15.9)$ & $6,295(16.0)$ & $716(15.0)$ & .07 \\
\hline Malignancy & $4,324(9.8)$ & $3,895(9.9)$ & $454(9.5)$ & .48 \\
\hline Weight loss & $1,720(3.9)$ & $983(2.5)$ & $750(15.7)$ & $<.001$ \\
\hline Valvular heart disease & $794(1.8)$ & $629(1.6)$ & $1,141(23.9)$ & $<.001$ \\
\hline Peripheral vascular disease & $485(1.1)$ & $189(0.5)$ & $314(6.6)$ & $<.001$ \\
\hline Hypothyroidism & $441(1.0)$ & $138(0.5)$ & $286(6.0)$ & $<.001$ \\
\hline Peptic ulcer disease & $383(0.9)$ & $338(0.9)$ & $45(0.9)$ & .63 \\
\hline Collagen vascular disease & $105(0.2)$ & $106(0.3)$ & $1(0.1)$ & .001 \\
\hline Acquired immune deficiency syndrome & $83(0.2)$ & $43(0.1)$ & $40(0.8)$ & $<.001$ \\
\hline Pulmonary vascular disease & $70.5(0.1)$ & $70(0.2)$ & $2(0.1)$ & .044 \\
\hline Coagulopathy & $61.7(0.1)$ & $51(0.1)$ & $13(0.3)$ & .02 \\
\hline Liver disease & $52(0.2)$ & $11(0.0)$ & $40(0.8)$ & $<.001$ \\
\hline Payer source & & & & $<.001$ \\
\hline Commercial insurance & 7,898 (17.9) & 6,964 (17.7) & $916(19.2)$ & \\
\hline Medicare & $23,782(53.9)$ & $21,147(54.0)$ & $2,536(53.1)$ & \\
\hline Medicaid & 7,324 (16.6) & $6,374(16.2)$ & $917(19.2)$ & \\
\hline Self pay & $1,985(4.5)$ & $1,888(4.8)$ & $119(2.5)$ & \\
\hline Other & $2,912(6.6)$ & $2,675(6.8)$ & $258(5.4)$ & \\
\hline No data available & $176(0.4)$ & $157(0.4)$ & $24(0.5)$ & \\
\hline
\end{tabular}

$\overline{\text { Values are number }(\%)}$ unless otherwise indicated.

* $P$ values for tracheostomy versus non-tracheostomy subjects.

\section{Human Subjects Protection}

The data used in this analysis represented a limited data set (ie, contained no direct patient identifiers) that was originally collected for non-research purposes. As such, this study was classified as exempt by the Washington University School of Medicine Human Research Protection Office (ie, did not involve human subjects) (HRPO 10-1190).

\section{Results}

\section{Characteristics of the Academic Medical Centers, Subjects, and Tracheostomy Practice}

We analyzed data from 44,124 subjects with the principal diagnosis of ARF, cared for in 102 academic medical centers (mean \pm SD $575 \pm 218.1$ licensed beds, 
Table 2. Outcomes of Adult Subjects With Acute Respiratory Failure Cared for in Academic Medical Centers

\begin{tabular}{|c|c|c|c|c|}
\hline & $\begin{array}{l}\text { All Subjects } \\
n=44,124\end{array}$ & $\begin{array}{c}\text { Non-tracheostomy } \\
\text { Subjects } \\
n=39,348\end{array}$ & $\begin{array}{c}\text { Tracheostomy } \\
\text { Subjects } \\
n=4,776\end{array}$ & $P$ \\
\hline Complications & $5,780(13.1)$ & $2,872(7.3)$ & $2,899(60.7)$ & $<.001$ \\
\hline Mortality & $11,781(26.7)$ & $10,781(27.4)$ & $984(20.6)$ & $<.001$ \\
\hline $\mathrm{ICU}$ stay, mean $\pm \mathrm{SD} \mathrm{d}$ & $8.5 \pm 10.5$ & $6.6 \pm 7.9$ & $24.3 \pm 20.7$ & $<.001$ \\
\hline Hospital stay, mean $\pm \mathrm{SD} d$ & $14.1 \pm 21.0$ & $11.3 \pm 19.8$ & $36.6 \pm 27.6$ & $<.001$ \\
\hline Total hospital costs, mean \pm SD $\$$ & $107,705 \pm 151,131$ & $86,118 \pm 107,771$ & $285,509 \pm 292,813$ & $<.001$ \\
\hline Discharge destination (survivors) & & & & $<.001$ \\
\hline Home (out-patient care) & $18,929(42.9)$ & $18,493(47.0)$ & $602(12.6)$ & \\
\hline Hospice & $1,014(2.3)$ & $944(2.4)$ & $72(1.5)$ & \\
\hline $\begin{array}{l}\text { In-patient setting (eg, acute care hospital, } \\
\text { rehabilitation, skilled nursing facility) }\end{array}$ & $23,959(54.3)$ & $19,753(50.2)$ & $4,069(85.2)$ & \\
\hline No data available & $221(0.5)$ & $157(0.4)$ & $33(0.7)$ & \\
\hline
\end{tabular}

Table 3. Outcomes of Adult Subjects With Acute Respiratory Failure Cared for in High Versus Low Resource Intensity Institutions

\begin{tabular}{|c|c|c|c|c|}
\hline & $\begin{array}{l}\text { All Subjects } \\
n=44,124\end{array}$ & $\begin{array}{c}\text { High Resource } \\
\text { Intensity } \\
n=4,435\end{array}$ & $\begin{array}{c}\text { Low Resource } \\
\text { Intensity } \\
n=39,689\end{array}$ & $P$ \\
\hline Complications, no. (\%) & $5,780(13.1)$ & $638(14.4)$ & $5,159(13.0)$ & .007 \\
\hline Mortality, no. (\%) & $11,781(26.7)$ & $1,357(30.6)$ & $10,438(26.3)$ & $<.001$ \\
\hline Tracheostomy, no. (\%) & $4,765(10.8)$ & $510(11.5)$ & 4,246 (10.7) & .13 \\
\hline $\mathrm{ICU}$ stay, mean $\pm \mathrm{SD} d$ & $8.5 \pm 10.5$ & $9.8 \pm 13.3$ & $8.4 \pm 19.9$ & $<.001$ \\
\hline Hospital stay, mean $\pm \mathrm{SD} d$ & $14.1 \pm 21.0$ & $17.5 \pm 20.0$ & $13.7 \pm 19.9$ & $<.001$ \\
\hline Total hospital costs, mean \pm SD $\$$ & $107,705 \pm 153,131$ & $182,106 \pm 263,986$ & $99,289 \pm 132,681$ & $<.001$ \\
\hline
\end{tabular}

$432.5 \pm 258.5$ subjects per center) over a 7-year period (2002 through 2008). Overall, 4,776 subjects (10.8\%) underwent tracheostomy (rate per center $10.8 \pm 3.0 \%$ ). The tracheostomy and non-tracheostomy subjects differed significantly with respect to several baseline variables: age, ethnicity, admission status, comorbidity, and payer source (Table 1). The tracheostomy subjects also had a higher morbidity rate, but a lower mortality rate than the nontracheostomy subjects. The tracheostomy subjects were also more resource-intensive to manage, as evidenced by longer ICU and hospital stay, higher total hospital costs, and greater likelihood of being discharged to an in-patient facility (long-term care facility, skilled nursing facility, rehabilitation facility) (Table 2).

\section{Differences in Tracheostomy Subject Outcomes Analyzed by Resource Intensity of the Care Environment}

We analyzed data from 102 university-affiliated academic medical centers. Twelve centers were designated as high resource intensity (ie, proportional expense ratio $\geq 1.25,4,435$ subjects, mean \pm SD subjects per center
$369.5 \pm 200.6$ ), and 90 centers as low resource intensity (ie, proportional expense ratio $<1.25,39,689$ subjects, mean \pm SD subjects per center $441.0 \pm 265.6)$. The high and low resource intensity institutions were similar with respect to number of licensed beds $(603.6 \pm 273.7$ vs $571.5 \pm 213.8, P=.64)$ and number of annual discharges of subjects with $\operatorname{ARF}(370 \pm 200$ vs $441 \pm 265, P=.44)$. The rates of tracheostomy did not differ, comparing these 2 environments $(510 / 4,435$ [11.5\%] in the high-resourceintensity institutions vs 4,246/39,689 [10.7\%] in the lowresource-intensity institutions, $P=.13$ ) (Table 3).

Variables found to be associated with mortality in univariate analysis were age, sex, probability of in-patient mortality, ICU stay, non-ICU stay, ethnicity, admission status (emergency vs non-emergency), payer source, presence of complications, and comorbidities present on admission (Table 4). In our multivariable analysis we found that being cared for in a high-resource-intensity environment was associated with increased risk of mortality (odds ratio $=1.11,95 \%$ CI $1.05-1.76$ ), while use of tracheostomy had no mortality effect (odds ratio $=0.99,95 \% \mathrm{CI}$ 0.92-1.06). However, the interaction term in our multivariable model demonstrated that tracheostomy subjects 
Table 4. Baseline Characteristics of Acute Respiratory Failure Tracheostomy Subjects in High Versus Low Resource Intensity Institutions

\begin{tabular}{|c|c|c|c|}
\hline & $\begin{array}{l}\text { High Resource } \\
\text { Intensity } \\
n=510\end{array}$ & $\begin{array}{c}\text { Low Resource } \\
\text { Intensity } \\
n=4,266\end{array}$ & $P$ \\
\hline Age, mean \pm SD y & $64.6 \pm 17.2$ & $59.9 \pm 15.9$ & $<.001$ \\
\hline Male & $243(47.8)$ & $2,231(52.3)$ & .06 \\
\hline Ethnicity & & & $<.001$ \\
\hline White & $256(50.3)$ & $2,611(61.2)$ & \\
\hline African American & $111(21.8)$ & $1,122(26.3)$ & \\
\hline Hispanic & $46(9.0)$ & $158(3.7)$ & \\
\hline Other & $96(18.8)$ & $371(8.7)$ & \\
\hline Admission status & & & $<.001$ \\
\hline Emergency & $350(68.6)$ & $2,755(64.6)$ & \\
\hline Urgent & $119(23.3)$ & $1,220(28.6)$ & \\
\hline Elective & $18(3.5)$ & $226(5.3)$ & \\
\hline Other or no data & $23(4.5)$ & $60(1.4)$ & \\
\hline \multicolumn{4}{|l|}{ Comorbidities } \\
\hline $\begin{array}{l}\text { Acquired immune deficiency } \\
\text { syndrome }\end{array}$ & $6(1.2)$ & $38(0.9)$ & .78 \\
\hline Anemia & $147(28.8)$ & $1,394(32.7)$ & .08 \\
\hline Chronic pulmonary disease & $198(38.8)$ & $1,974(46.3)$ & .002 \\
\hline Coagulopathy & $76(14.9)$ & $657(15.4)$ & .80 \\
\hline Collagen vascular disease & $22(4.3)$ & $132(3.1)$ & .17 \\
\hline Congestive heart failure & $179(35.1)$ & $1,505(35.3)$ & .95 \\
\hline Diabetes & $109(21.4)$ & $1,288(30.2)$ & $<.001$ \\
\hline Fluid and electrolyte disorders & $259(50.8)$ & $2,546(59.7)$ & $<.001$ \\
\hline Hypertension & $228(44.9)$ & $2,073(48.6)$ & .12 \\
\hline Hypothyroidism & $38(7.4)$ & $375(8.8)$ & .34 \\
\hline Liver disease & $23(4.5)$ & $196(4.6)$ & .89 \\
\hline Malignancy & $90(17.6)$ & $367(8.6)$ & $<.001$ \\
\hline Neurological disorder & $137(26.9)$ & $1,079(25.3)$ & .48 \\
\hline Peptic ulcer disease & $2(0.4)$ & $17(0.4)$ & .79 \\
\hline Peripheral vascular disease & $22(4.3)$ & $188(4.4)$ & .99 \\
\hline $\begin{array}{l}\text { Psychiatric disorder } \\
\text { (depression, psychoses) }\end{array}$ & $59(11.6)$ & $657(15.4)$ & .02 \\
\hline Pulmonary vascular disease & $48(9.4)$ & $447(10.5)$ & .50 \\
\hline Renal failure & $79(15.5)$ & $695(16.3)$ & .67 \\
\hline Valvular heart disease & $52(10.2)$ & $375(8.8)$ & .32 \\
\hline Weight loss & $84(16.5)$ & $947(22.2)$ & .004 \\
\hline Payer source & & & .20 \\
\hline Commercial insurance & $108(21.2)$ & $810(19.0)$ & \\
\hline Medicare & $281(55.1)$ & $2,252(52.8)$ & \\
\hline Medicaid & $84(16.5)$ & $836(19.6)$ & \\
\hline Other & $37(7.2)$ & $367(8.6)$ & \\
\hline
\end{tabular}

$\overline{\text { Values are number }(\%)}$ unless otherwise indicated.

managed in high-resource-intensity settings were at increased risk of death (odds ratio $=1.10,95 \%$ CI $1.04-1.17$ ) (Table 5). Results were comparable whether resource intensity was modeled as a dichotomous or continuous variable (see the supplementary materials at http://www. rcjournal.com).
We explored possible reasons that might underlie our finding of an unfavorable relationship between resource intensity and outcome for tracheostomy subjects. To examine whether this relationship might be the result of early discharge of subjects at high risk of mortality from lowresource-intensity settings, we analyzed post-hospital discharge destination. We found that there was not a statistically significant trend toward high-resource-intensity settings being more likely to transfer subjects to other in-patient institutions following discharge (skilled nursing facilities 115/406 [28.5\%], intermediate care facilities 156/ 406 [38.5\%], rehabilitation centers 72/406 [17.7\%], general hospitals 36/406 [8.9\%], other environments 26/406 [6.4\%], with low-resource-intensity settings being more likely to discharge subjects to home) (Table 6).

We considered whether the increased risk of mortality we observed may be related to an increased number or type of complications in high-resource-intensity settings. However, we found that the average number of complications per subject $(1.54 \pm 0.65$ in low resource intensity vs $1.60 \pm 0.22$ in high resource intensity, $P=.13$ ), the incidence of complications per subject per day $(0.036 \pm 0.065$ in low resource intensity vs $0.034 \pm 0.072$ in high resource intensity, $P=.11$ ), and profile of complications $(P>.05$ for all $)$ were similar.

Finally, we examined the relationship between complications and mortality. We found that mortality for tracheostomy subjects experiencing complications was greater in the high-resource-intensity than in the low-resource-intensity environments: 95/313 (30.3\%) vs 552/2587 (21.3\%) $(P<.001)$. In contrast, mortality rates in the tracheostomy subjects with no complications were similar: 41/197 $(20.8 \%)$ vs $297 / 1679(17.7 \%)(P=.33)$.

\section{Discussion}

The relationship between resource expenditure and outcome in healthcare settings is variable. ${ }^{23-27}$ Studies have found that higher expenditure was associated with lower 30-day mortality, 1-year mortality, and major cardiac events among patients presenting to acute care hospitals with a variety of diagnoses..$^{24,25}$ In contrast, Garland et al reported that variation in discretionary resource use among individual medical intensivists did not translate into differences in in-hospital mortality or stay. ${ }^{23}$ Given the variability in tracheostomy utilization and the resource consumption associated with providing care to patients undergoing this procedure, we examined the effects of resource expenditure in this setting. Consistent with the findings of prior studies, the resource expenditure associated with providing care to this patient population was significant, with total aggregate costs exceeding $\$ 1.6$ billion. ${ }^{1,3,4}$ Though representing only $10 \%$ of our sample, tracheostomy subjects accounted for $28.4 \%$ of these costs (collectively, $\$ 463$ million). We 
Table 5. Univariate Analysis and Odds Ratios for Variables Included in Logistic Regression Model for Mortality

\begin{tabular}{|c|c|c|c|c|c|}
\hline & \multicolumn{2}{|c|}{ Univariate Analysis } & \multirow[b]{2}{*}{$P$} & \multicolumn{2}{|c|}{ Multivariable Analysis } \\
\hline & $\begin{array}{c}\text { Non-survivors } \\
n=11,798\end{array}$ & $\begin{array}{c}\text { Survivors } \\
n=32,326\end{array}$ & & $\begin{array}{l}\text { Odds } \\
\text { Ratio }\end{array}$ & $95 \% \mathrm{CI}$ \\
\hline \multicolumn{6}{|l|}{ Variables significant in multivariable model } \\
\hline Complications $(\geq 1)$ & $1,439(12.2)$ & $4,368(13.5)$ & $<.001$ & 1.18 & $1.10-1.28$ \\
\hline High resource intensity institution & $1,357(11.5)$ & $3,073(9.5)$ & $<.001$ & 1.11 & $1.05-1.76$ \\
\hline Tracheostomy $\times$ High resource intensity institution & $165(1.4)$ & $453(1.4)$ & .88 & 1.10 & $1.04-1.17$ \\
\hline Expected Death Rate, mean \pm SD $\%$ & $37.5 \pm 20.0$ & $23.8 \pm 14.4$ & $<.001$ & 1.03 & $1.03-1.04$ \\
\hline Age, mean \pm SD y & $65.3 \pm 21.7$ & $59.9 \pm 17.9$ & $<.001$ & 1.01 & $1.01-1.01$ \\
\hline $\mathrm{ICU}$ stay, mean $\pm \mathrm{SD} \mathrm{d}$ & $9.0 \pm 12.3$ & $8.4 \pm 10.9$ & $<.001$ & 1.01 & $1.00-1.01$ \\
\hline Non-ICU stay, mean \pm SD d & $3.0 \pm 11.4$ & $6.5 \pm 11.7$ & $<.001$ & 0.93 & $0.92-0.93$ \\
\hline Admission status (emergency, non-emergency) & $8,223(69.7)$ & $23,684(73.2)$ & $<.001$ & 0.91 & $0.86-0.96$ \\
\hline \multicolumn{6}{|l|}{ Comorbidities } \\
\hline Solid tumor with metastasis & $1,392(11.8)$ & $906(2.8)$ & $<.001$ & 2.18 & $1.98-2.41$ \\
\hline Coagulopathy & $2,159(18.3)$ & $2,880(8.9)$ & $<.001$ & 1.48 & $1.38-1.59$ \\
\hline Solid tumor without metastasis & $802(6.8)$ & $1,229(3.8)$ & $<.001$ & 1.45 & $1.31-1.60$ \\
\hline Liver disease & $932(7.9)$ & $1,585(4.9)$ & $<.001$ & 1.42 & $1.29-1.57$ \\
\hline Lymphoma & $354(3.0)$ & $388(1.2)$ & $<.001$ & 1.44 & $1.22-1.70$ \\
\hline Rheumatoid arthritis/collagen vascular disease & $460(3.9)$ & $971(3.0)$ & $<.001$ & 1.36 & $1.20-1.54$ \\
\hline Fluid and electrolyte abnormality & $6,335(53.7)$ & $14,140(43.7)$ & $<.001$ & 1.14 & $1.08-1.20$ \\
\hline Renal failure & $2,218(18.8)$ & $5,597(17.3)$ & $<.001$ & 1.14 & $1.07-1.22$ \\
\hline Paralysis & $448(3.8)$ & $1,650(5.1)$ & $<.001$ & 0.87 & $0.78-0.97$ \\
\hline Congestive heart failure & $4,070(34.5)$ & $11,907(36.8)$ & $<.001$ & 0.87 & $0.82-0.92$ \\
\hline Obesity & $743(6.3)$ & $3,656(11.3)$ & $<.001$ & 0.84 & $0.77-0.92$ \\
\hline Alcohol abuse & $755(6.4)$ & $3,170(9.8)$ & $<.001$ & 0.83 & $0.75-0.91$ \\
\hline Deficiency anemias & $2,394(20.3)$ & $8,541(26.4)$ & $<.001$ & 0.75 & $0.71-0.79$ \\
\hline Depression & $708(6.0)$ & $3,494(10.8)$ & $<.001$ & 0.75 & $0.69-0.82$ \\
\hline Hypertension & $5,403(45.8)$ & $17,795(55.0)$ & $<.001$ & 0.75 & $0.71-0.79$ \\
\hline Drug abuse & $365(3.1)$ & $2,847(8.8)$ & $<.001$ & 0.64 & $0.58-0.74$ \\
\hline Psychoses & $354(3.0)$ & $2,491(7.7)$ & $<.001$ & 0.63 & $0.56-0.71$ \\
\hline Chronic pulmonary disease & $4,377(37.1)$ & $17,083(52.8)$ & $<.001$ & 0.63 & $0.60-0.66$ \\
\hline \multicolumn{6}{|l|}{ Variables not significant in multivariable model } \\
\hline Payer source (Medicaid) & $1,380(11.7)$ & $4,885(15.1)$ & $<.001$ & 1.04 & $0.97-1.12$ \\
\hline Tracheostomy & $979(8.3)$ & $3,785(11.7)$ & $<.001$ & 0.99 & $0.92-1.06$ \\
\hline Female & $5,568(47.2)$ & $15,919(49.2)$ & $<.001$ & 0.97 & $0.92-1.02$ \\
\hline Ethnicity non-white & $4,613(39.1)$ & $13,880(42.9)$ & $<.001$ & 0.96 & $0.91-1.01$ \\
\hline \multicolumn{6}{|l|}{ Comorbidities } \\
\hline Valvular heart disease & $1,226(10.4)$ & $2,912(9.0)$ & $<.001$ & 1.00 & $0.93-1.09$ \\
\hline Diabetes (without complications) & $2,667(22.6)$ & $8,218(25.4)$ & $<.001$ & 0.95 & $0.90-1.01$ \\
\hline Chronic blood loss anemia & $189(1.6)$ & $421(1.3)$ & .01 & 0.95 & $0.79-1.14$ \\
\hline Diabetes (with complications) & $519(4.4)$ & $1,714(5.3)$ & $<.001$ & 0.94 & $0.84-1.05$ \\
\hline
\end{tabular}

limited our analysis to subjects with the principal diagnosis of ARF, assuming that indication for tracheostomy would be more consistent across institutions than if all individuals, irrespective of principal diagnosis, were included. Nonetheless, individuals in this study were complex, had substantial comorbidities, and had a death rate of $>25 \%$. The tracheostomy subjects had a lower mortality rate than the non-tracheostomy subjects. Whether this resulted from practitioners targeting tracheostomy to individuals most likely to survive the acute episode of illness, or whether subjects undergoing tracheostomy survived until transfer to a non-acute hospital setting and then succumbed cannot be discerned from this analysis. Furthermore, the tracheostomy subjects had a higher complication rate, longer ICU and hospital stay, and higher overall costs than the non-tracheostomy subjects. We based institutional resource intensity on risk-adjusted estimates of costs associated with providing care. In contrast both to prior studies and our stated hypothesis, we found an inverse relationship between resource expenditure and outcome. ${ }^{23-27}$ 


\section{High Resource Utilization Does Not Affect Mortality in ARF Patients With Tracheostomy}

Table 6. Outcomes of Acute Respiratory Failure Tracheostomy Subjects in High Versus Low Resource Intensity Institutions

\begin{tabular}{|c|c|c|c|}
\hline & $\begin{array}{l}\text { High Resource Intensity } \\
\qquad n=510\end{array}$ & $\begin{array}{l}\text { Low Resource Intensity } \\
\qquad n=4,266\end{array}$ & $P$ \\
\hline Complications, no. (\%) & $313(61.4)$ & $2,585(60.6)$ & .79 \\
\hline Mortality, no. (\%) & $136(26.7)$ & $849(19.9)$ & $<.001$ \\
\hline $\mathrm{ICU}$ stay, mean $\pm \mathrm{SD} \mathrm{d}$ & $28.1 \pm 26.4$ & $23.9 \pm 18.8$ & .04 \\
\hline Hospital stay, mean $\pm \mathrm{SD} d$ & $44.8 \pm 38.1$ & $35.6 \pm 30.5$ & $<.001$ \\
\hline Total hospital costs, mean \pm SD $\$$ & $166,567 \pm 145,410$ & $95,972 \pm 78,557$ & $<.001$ \\
\hline Discharge destination (survivors), no. (\%) & & & .057 \\
\hline Home (out-patient care) & $96(18.9)$ & $1,041(24.4)$ & \\
\hline Hospice & $4(0.8)$ & $64(1.5)$ & \\
\hline $\begin{array}{l}\text { In-patient setting (eg, acute care hospital, } \\
\text { rehabilitation, skilled nursing) }\end{array}$ & $406(79.7)$ & $3,123(73.2)$ & \\
\hline No data available & $2(0.5)$ & $34(0.8)$ & \\
\hline
\end{tabular}

Specifically, compared with low-resource-intensity settings, subjects managed in high-resource-intensity institutions had higher in-hospital mortality, and tracheostomy was associated with as great as $17 \%$ higher likelihood of death. Thus, not only did institutions appear to differ with respect to the costs of care, the additional resources expended in high-resource-intensity settings did not appear to positively affect outcome.

We took 2 complementary approaches to performing our analysis: one in which the institutions were stratified into high and low resource-intensity categories, and the second in which resource intensity was modeled as a continuous variable. The intent of this latter approach was to compensate for any bias that discrete modeling of this variable might introduce (ie, arbitrary assignment of the high and low designation). Both methodologies produced comparable findings. We explored reasons that might underlie the observed relationship between resource intensity and mortality. We reasoned that low-resource-intensity environments may appear to have more favorable outcomes for tracheostomy subjects because of more timely transfer to other in-patient settings (such as skilled nursing or longterm weaning facilities). Deaths occurring in such settings would not be captured as in-patient mortality. However, not only were the low and high resource-intensity environments similar with respect to post-hospital discharge destinations, there was a trend for low-resource-intensity environments to more commonly discharge subjects to home. We similarly reasoned that higher rates of complications may underlie the greater resource expenditure and higher mortality in high-resource-intensity environments. Again, we found that low and high-resource-intensity environments were similar with respect to the incidence and total number of complications. However, mortality for the tracheostomy subjects who had complications was greater in the high-resource-intensity environments, suggesting a limited ability to rescue subjects following the development of complications. Potentially, these findings result from important differences in the patient populations between the low and high resource-intensity settings, which were not adequately adjusted for in our analysis. Thus, high-resource-intensity institutions may have managed subjects of greater acuity, explaining more common discharge to in-patient settings and higher mortality following the occurrence of complications. Accordingly, one must be circumspect in these interpretations.

Recent studies examining tracheostomy implementation have focused on specific outcomes such as duration of mechanical ventilation, ICU stay, and sedative use. ${ }^{18-20}$ One interpretation of the current analysis is that it is difficult to disaggregate the use of tracheostomy from other facets of care. ICUs that apply differing approaches to ventilator weaning, sedation, venous thromboembolism prophylaxis, and other interventions may likewise differ significantly in the manner in which they apply tracheostomy. ${ }^{32-34}$ Furthermore, post-tracheostomy care (approaches to phonation, nutrition, re-conditioning, and decannulation) might differ among institutions and influence outcome. ${ }^{9}$ Our data set did not provide us with information on how care was provided, which limits our ability to identify factors and behaviors underlying the outcomes observed. We lack knowledge of factors that might prompt the decision for tracheostomy (eg, repetitive extubation failure, lack of progress on sequential weaning trial attempts). An appreciable proportion of the subjects described in this study had Medicare or Medicaid as a payer source; reimbursement considerations and similar non-clinical factors may have influenced the decision for tracheostomy in some contexts. Determining these factors (eg, through site visits or inventory of practices) and translating approaches effective at well-performing institutions to those that perform suboptimally would be one approach to enhancing patient safety and quality.

Our investigation has several additional limitations. The University Health Systems Consortium is a network of academic medical centers that may be self-selecting in 
terms of commitment to quality-assurance initiatives and other attributes. The severity of illness and complexity of patients cared for in these institutions may differ substantially from other settings (particularly non-academic institutions), limiting the ability to generalize these findings. In addition, this analysis is based on administrative coding data. These codes are designed to enable billing and are not necessarily a direct reflection of medical care received. Inaccuracy is potentially introduced through code attribution by non-clinical personnel. These shortcomings are partly offset by the fact that such codes are standardized, appropriately updated, and reasonably specific. Their availability in large numbers has the potential to "drown out" statistical noise and error. Also, our analysis might be confounded by the possibility that patient complexity is directly related to both resource intensity and likelihood of poor outcome. We structured our multivariable model so as to control for this possibility, stratifying hospitals based on previously validated risk-adjusted estimates of costs associated with providing care. ${ }^{28-30}$ Though this method was uniformly applied to all institutions in our sample, it might not adequately account for all factors critical to outcome. For instance, geography and setting (ie, urban, suburban) may be an influential demographic factor that we did not capture.

These and other types of limitations might be further magnified by the lack of inclusion of physiologic data (eg, Acute Physiology and Chronic Health Evaluation score) that might have allowed us to further refine our estimates of illness acuity. Similarly, while we identified a large number of complications, many were defined in the context of the postoperative state. Thus, some important adverse events might go undetected. Finally, we undertook an institution-wide assessment of tracheostomy practice, focusing on patients with ARF. This approach ignores the very real differences in tracheostomy utilization and critical care practice that might exist within specific hospital domains (eg, comparing surgical and medical ICUs), and our findings might not be reflective of other disease processes (eg, acute neurological insult or polytrauma).

\section{Conclusions}

We were unable to demonstrate a positive relationship between resource expenditure and outcome in ARF patients managed with tracheostomy. Given the exploratory nature of this study, one must be circumspect about any conclusions drawn. Our analysis does suggest several potential avenues of further inquiry. Among these are investigations that focus on identifying clinically beneficial strategies related to tracheostomy patient selection, timing, technique, and post-procedure management, and assessing the cost-effectiveness of these strategies when applied across diverse clinical environments.

\section{REFERENCES}

1. Dewar DM, Kurek CJ, Lambrinos J, Cohen IL, Zhong Y. Patterns in costs and outcomes for patients with prolonged mechanical ventilation undergoing tracheostomy: an analysis of discharges under diagnosis-related group 483 in New York State from 1992 to 1996. Crit Care Med 2003;27(12):2640-2647.

2. Heffner JE. The role of tracheotomy in weaning. Chest 2001;120(6): 477S-481S.

3. Frutos-Vival F, Esteban A, Apezteguía C, Anzueto A, Nightingale P, Gonzalez M, et al. Outcome of mechanically ventilated patients who require a tracheostomy. Crit Care Med 2005;33(2):290-298.

4. Cox CE, Carson SS, Holmes GM, Howard A, Carey TS. Increase in tracheostomy for prolonged mechanical ventilation in North Carolina, 1993-2002. Crit Care Med 2004;32(11):2219-2226.

5. Freeman BD, Borecki IB, Coopersmith CM, Buchman TB. Relationship between tracheostomy timing and duration of mechanical ventilation in critically ill patients. Crit Care Med 2005;33(11):2513-2520.

6. Cox CE, Martinu T, Sathy S, Clay AS, Chia J, Gray AL, et al. Expectations and outcomes of prolonged mechanical ventilation. Crit Care Med 2009;37(11):2888-2894.

7. Kurek CJ, Cohen IL, Lambrinos J, Minatoya K, Booth FV, Chalfin DB. Clinical and economic outcome of patients undergoing tracheostomy for prolonged mechanical ventilation in New York State during 1993: analysis of 6,353 cases under diagnosis-related group 483. Crit Care Med 1997;25(6):983-988.

8. Merrill CH, Elixhauser A; US Department of Health \& Human Services; Agency for Healthcare Research and Quality. HCUP Fact Book \#7: Procedures in US Hospitals, 2003. AHRQ Publication no. 06-0039. http://archive.ahrq.gov/data/hcup/factbk7. Accessed August 23, 2013.

9. Freeman BD, Morris PE. Concise Definitive Review: Tracheostomy practice in adults with acute respiratory failure. Crit Care Med 2012; 40(10):2890-2896.

10. Mirski MA, Pandian V, Bhatti N, Haut E. Safety, efficacy, and cost-effectiveness of a multidisciplinary percutaneous tracheostomy program. Crit Care Med 2012;40(6):1827-1834.

11. Freeman BD. Implementation of a specialized tracheostomy team as a strategy for quality improvement. Crit Care Med 2012;40(6):1980-1981.

12. Rodriguez JL, Steinberg SM, Luchetti FA, Gibbons KJ, Taheri PA, Flint LM. Early tracheostomy for primary airway management in the surgical critical care setting. Surgery 1990;108(4):655-659.

13. Rumbak MJ, Newton M, Truncale T, Schwartz SK, Adams JW, Hazard PB. A prospective randomized, study comparing early percutaneous dilational tracheotomy to prolonged translaryngeal intubation (delayed tracheotomy) in critically ill medical patients. Crit Care Med 2004;32(1):1689-1694.

14. Brook AD, Sherman G, Malen J, Kollef MH. A comparison of earlyvs late-tracheostomy in patients requiring prolonged mechanical ventilation. Am J Crit Care 2000;9(5):352-359.

15. Blot F, Guiguet M, Antoun S, Leclercq B, Nitenberg G, Escudier B. Early tracheotomy in neutropenic, mechanically ventilated patients: rationale and results of a pilot study. Support Care Can 1995;3(5): 291-296.

16. Boynton JH, Hawkins K, Eastridge BJ, O'Keefe GE. Tracheostomy timing and the duration of weaning in patients with acute respiratory failure. Crit Care 2004;8:R261-R267.

17. Barquist E, Amortegui J, Hallal A, Giannotti G, Whinney R, Alzamel H, MaCleod J. Tracheostomy in ventilator dependent trauma patients: a prospective, randomized intention-to-treat study. J Trauma 2006;60(1):91-97.

18. Terragni PP, Antonelli M, Fumagalli R, Faggiano C, Berardino M, Pallavicini FB et al. Early vs late tracheotomy for prevention of pneumonia in mechanically ventilated adult ICU patients. JAMA 2010;303(15):1483-89. 
19. Young, D. Early tracheostomy reduces sedative use but does not affect mortality. Brussels: 29th International Symposium on Intensive Care and Emergency Medicine; March 26, 2009.

20. Trouillet JL, Luyt CE, Guiguet M, Ouattara A, Valsser E, Makri R, et al. Early percutaneous tracheotomy versus prolonged intubation of mechanically ventilated patients after cardiac surgery. Ann Int Med 2011;154(6):373-383.

21. Nathens AB, Rivara FP, Mack CD, Rubenfeld GD, Wang J, Jurkovich GJ et al. Variations in the rates of tracheostomy in the critically ill trauma patient. Crit Care Med 2006;34(12):2919-2924.

22. Freeman BD, Kennedy C, Coopersmith CM, Buchman TB. Examination of non-clinical factors affecting tracheostomy practice in an academic surgical intensive care unit. Crit Care Med 2009;37(12): 3070-3078.

23. Garland A, Shamon Z, Baron J, Conners AF. Physician-attributable differences in intensive care unit costs. Am J Respir Crit Care Med 2006;174(11):1206-1210.

24. Stukel TA, Fisher ES, Alter DA, Guttmann A, Ko DT, Fung K et al. Association of hospital spending intensity with mortality and readmission rates in Ontario hospitals. JAMA 2012;307(10):1037-1045.

25. Chen LM, Jha AK, Guterman S, Ridgway AB, Orav J, Epstein AM. Hospital cost of care, quality of care, and readmission rates: penny wise and pound foolish? Arch Int Med 2012;170(4):340-346.

26. Barnato AE, Chang CC, Farrell MH, Lave JR, Roberts MS, Angus DC. Is survival better at hospitals with higher 'end of life' treatment intensity? Med Care 2010;48(2):125-132.
27. Romley JA, Jena AB, Goldman DP. Hospital spending and inpatient mortality: evidence from California. An observational study. Ann Int Med 2011;154(3):160-167.

28. Iezzoni LI, editor. Risk adjustment for measuring healthcare outcomes, 3rd edition. Chicago: ACHE/Health Administration Press; 2003.

29. Kim DH, Daskalakis C, Lee AN, Adams S, Hohmann S, Silvestry $\mathrm{SC}$ et al. Racial disparity in the relationship between hospital volume and mortality among patients undergoing coronary artery bypass grafting. Ann Surg 2009;248(5):886-892.

30. Bankowitz RA, Neikirk MA, Juris A. A UHC clinical database analyst reference manual. Oak Brook, IL: University Healthsystems Consortium; 2000.

31. Elixhauser A, Steiner C, Harris DR, Coffey RM. Comorbidity measures for use of administrative data. Med Care 1998;36(1):3-5.

32. Esteban A, Frutos F, Tobin MJ, Alía I, Solsona JF, Valverdú I et al. A comparison of four methods of weaning patients from mechanical ventilation. N Engl J Med 1995;332(6):345-350.

33. Esteban A, Alía I, Tobin MJ, Gil A, Gordo F, Vallverdú I et al. Effect of spontaneous breathing trial duration on outcomes of attempts to discontinue mechanical ventilation. Am J Respir Crit Care Med 1999;159(2):512-519.

34. Kress JP, Pohlman AS, O'Connor MF, Hall JB. Daily interruption of sedative infusions in critically ill patients undergoing mechanical ventilation. N Engl J Med 2000;342(20):1471-1477.

This article is approved for Continuing Respiratory Care Education credit. For information and to obtain your CRCE

(free to AARC members) visit www.rcjournal.com

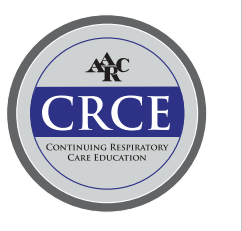

